\title{
Valores de chumbo inorgânico em suplementos minerais comercializados no estado de São Paulo
}

\author{
Values of lead in mineral supplements commercialized \\ in São Paulo State, Brazil
}

Wilmar Sachetin Marçal, ${ }^{*}$ Marcos Roberto Lopes do Nascimento, ${ }^{* \star}$ Maíra Fortes Salomão,,** Denis Rodrigues Prata***

\begin{abstract}
Resumo
Foi realizada uma pesquisa quantificando o elemento chumbo em diferentes formulações, comercializadas no Estado de São Paulo. O metal pesado foi determinado pela técnica de espectrofotometria de absorção atômica por plasma de indução acoplada. Considerando como parâmetro de comparação a referência de Maletto (1986), em 40 diferentes marcas analisadas, 31 apresentaram níveis de chumbo inorgânico superiores ao limite máximo aceitável que é de 10 ppm.
\end{abstract}

Palavras-chave: metais pesados, chumbo, suplementos minerais, bovinos.

\begin{abstract}
We measured lead concentrations in samples of mineral mixtures commercialized in cities of São Paulo state. Lead content was determined by inductively coupled plasma atomic emission spectrometry. Considering maximum concentrations recommended by Maletto (1986), of the 40 analyzed samples, 31 had values greater than $10 \mathrm{ppm}$.
\end{abstract}

Keywords: heavy metals, lead, mineral supplements, cattle.

Os recentes episódios da Síndrome da Vaca Louca e Febre Aftosa na Europa colocou o Brasil numa condição especial como exportador de carne. Por isso, monitorar as fontes de matérias-primas que passarão a compor a nutrição mineral dos bovinos, é neste momento de fundamental importância para a pecuária brasileira. Nesse aspecto, a proposta deste estudo foi investigar a presença do elemento chumbo nos suplementos minerais, visando a dar alguma contribuição às ações de rastreabilidade e produção orgânica de bovinos no Brasil. Até então, não havia trabalho desta natureza, considerando as formulações mais comercializadas no Estado de São Paulo, onde existem 1376 diferentes marcas (Marçal et al., 1998).

O chumbo quando presente em suplementos alimentares para animais pode causar alterações orgânicas importantes, modificando a performance dos animais (Maletto, 1986; Silva, 1993; Association of American Feed Control Officials Incorporated, 2001), podendo caracterizar uma cadeia alimen- tar comprometida, atingindo os bovinos e, por conseguinte, o homem (Maletto, 1986; Junqueira, 1993; Marçal et al., 2001), representando riscos à saúde pública pelo consumo de produtos e subprodutos de origem animal comprometidos (Allen, 1992; Campos Neto e Marçal, 1996). Assim exposto, o objetivo deste trabalho foi investigar a presença de chumbo em diferentes suplementos minerais comercializados no Estado de São Paulo.

As amostras de sal mineral foram colhidas em estabelecimentos comerciais e propriedades rurais, sendo acondicionadas em recipientes de plástico transparente, previamente identificadas, com aproximadamente 200 gramas de cada diferente marca de produto. As análises foram efetuadas no Laboratório da Comissão Nacional de Energia Nuclear (CNEN) de Poços de Caldas, Minas Gerais. A metodologia de análise empregada baseia-se na descrição de Eaton et al. (1995). O limite mínimo de determinação de chumbo no método é de 1,5 ppm.

\footnotetext{
* Médico-veterinário e professor associado do Departamento de Clínicas Veterinárias da Universidade Estadual de Londrina, Caixa Postal nº 6001, CEP 86051-970, Londrina, PR. E-mail: wilmar@uel.br

** Químico da Comissão Nacional de Energia Nuclear, Poços de Caldas, MG.

*** Acadêmico de medicina-veterinária da Universidade Estadual de Londrina. Bolsista PIBIC/CNPq.
} 
Os resultados encontrados na análise das formulações minerais demonstram que das 40 amostras investigadas, 31 tiveram valores que extrapolaram o limite máximo aceitável de 10 ppm estabelecido por Maletto (1986), representando mais de $77 \%$ das formulações analisadas. O maior valor encontrado, ou seja, 284 ppm, refere-se a uma formulação mi-

Tabela 1 - Resultados (média e desvio-padrão) da quantificação do elemento chumbo inorgânico em diferentes marcas de sal mineral, comercializadas em alguns municípios no Estado de São Paulo, 2003

\begin{tabular}{|c|c|c|}
\hline no da amostra & Município & $\begin{array}{c}\text { Valores de chumbo } \\
\text { em ppm }\end{array}$ \\
\hline 01 & Araçatuba & $4,3 \pm 0,4$ \\
\hline 02 & Avaré & $3,8 \pm 0,4$ \\
\hline 03 & Avaré & $15,4 \pm 1,3$ \\
\hline 04 & Avaré & $35,7 \pm 2,8$ \\
\hline 05 & Avaré & $3,4 \pm 0,3$ \\
\hline 06 & Bastos & $94 \pm 3,7$ \\
\hline 07 & Bastos & $30 \pm 1,2$ \\
\hline 08 & Batatais & $20,2 \pm 1,0$ \\
\hline 09 & Bauru & $9,9 \pm 0,8$ \\
\hline 10 & Birigüi & $13,7 \pm 0,7$ \\
\hline 11 & Birigüi & $18,2 \pm 0,9$ \\
\hline 12 & Campinas & $146 \pm 7,0$ \\
\hline 13 & Castilho & $5,6 \pm 0,4$ \\
\hline 14 & Fernandópolis & $16,0 \pm 0,8$ \\
\hline 15 & General Salgado & $26,2 \pm 1,3$ \\
\hline 16 & lepê & $38 \pm 1,8$ \\
\hline 17 & Jaboticabal & $112 \pm 5,1$ \\
\hline 18 & Jaboticabal & $17 \pm 0,8$ \\
\hline 19 & Jaboticabal & $30 \pm 1,0$ \\
\hline 20 & Jales & $16 \pm 1,4$ \\
\hline 21 & Martinópolis & $28,2 \pm 1,4$ \\
\hline 22 & Martinópolis & $19,1 \pm 0,9$ \\
\hline 23 & Martinópolis & $284 \pm 14$ \\
\hline 24 & Mogi mirim & $50,8 \pm 2,5$ \\
\hline 25 & Piracicaba & $28,7 \pm 1,4$ \\
\hline 26 & Presidente Bernardes & $5,0 \pm 0,4$ \\
\hline 27 & Presidente Bernardes & $3,1 \pm 0,2$ \\
\hline 28 & Presidente Bernardes & $27,6 \pm 1,3$ \\
\hline 29 & Presidente Epitácio & $8,0 \pm 0,9$ \\
\hline 30 & Presidente Prudente & $44 \pm 1,5$ \\
\hline 31 & Presidente Prudente & $79 \pm 3,4$ \\
\hline 32 & Ribeirão Preto & $3,0 \pm 0,3$ \\
\hline 33 & Ribeirão Preto & $134 \pm 6,0$ \\
\hline 34 & Ribeirão Preto & $37 \pm 1,6$ \\
\hline 35 & Sales Oliveira & $49 \pm 1,9$ \\
\hline 36 & Santo Anastácio & $156 \pm 7,6$ \\
\hline 37 & Santo Anastácio & $141 \pm 6,2$ \\
\hline 38 & São Paulo & $34,1 \pm 1,7$ \\
\hline 39 & Taciba & $43,8 \pm 2,2$ \\
\hline 40 & Taciba & $45 \pm 2,1$ \\
\hline
\end{tabular}

neral colhida na cidade de Martinópolis, importante pólo pecuário e comercial do Estado de São Paulo.

Nestas investigações sobre o chumbo utilizaram-se as formulações minerais já misturadas, porque não foi possível separar as matérias-primas para investigar cada um de seus componentes. A presença do chumbo pode ser originada de fontes de matérias-primas de macro e também de microminerais (Marçal et al., 2001). Espera-se, a partir desses resultados, considerados até mesmo como uma triagem, ser possível rastrear o destino das formulações comprometidas e monitorar a sanidade dos bovinos. Além disso, se as respectivas indústrias não aprimorarem o controle de qualidade e pureza de matérias-primas usadas na composição de suas formulações, o crescimento da comercialização desses sais levará a presença de mais contaminantes na alimentação animal, originando barreiras econômicas e sanitárias e causando queda no competitivo mercado comercial, sobretudo nas exportações.

\section{Referências}

ALLEN, J. D. Minerals in animal feed. Industrial Minerals, London, $\mathrm{n}$. 292, p. 35-39, 1992.

ASSOCIATION OF AMERICAN FEED CONTROL OFFICIALS INCORPORATED. Official guidelines for contaminant levels permitted in mineral feed ingredients. Indiana, 2001.

CAMPOS NETO, O.; MARÇAL W. S. Os fosfatos na nutrição mineral de ruminantes. Revista dos Criadores, São Paulo, n. 793, p. 8-10, 1996.

EATON, C. L. S.; GREENBERG, A. E.; TRUSSELL, R. R. (Eds.). Standard methods for the examination of water and wastewater. Washington: APHA, 1995.

JUNQUEIRA, O. M. Metais pesados contaminam carne. Avicultura \& Suinocultura Industrial, São Paulo, n. 38, p. 27-29, 1993.

MALETTO, S. Correlação da nutrição mineral e a sanidade. In: SEMINÁRIO SOBRE NUTRIÇÃO MINERAL, 1986, São Paulo. Anais... São Paulo, 1986. p. 38.

MARÇAL, W. S. et al. Lead concentration in mineral salt mixtures used in beef cattle food supplementation in Brazil. Veterinary Archiv, Croacia, v. 69 , n. 6, p. 349-355, 1999.

MARÇAL, W. S. et al. Concentration of lead in mineral salt mixtures used as supplements in cattle food. Experimenthal and Toxicologic Pathology, Jena, v. 53, p. 7-9, 2001.

MARÇAL, W. S.; CAMPOS NETO, O.; NASCIMENTO, M. R. L. Valores sangüíneos de chumbo em bovinos Nelore suplementados com sal mineral naturalmente contaminado por chumbo. Ciência Rural, Santa Maria, v. 28, n. 1, p. 53-57, 1998.

ROSA, I. V. Fosfato natural como suplemento de fósforo para bovinos. In: VALLE, E.R. et al. Coletânea de seminários técnicos 1986/88. Campo Grande: Embrapa, 1989, p. 59.

SILVA, S. Plano de ação fiscal sobre fosfato de rocha e outros. Brasília, DF, Ministério da Agricultura, do Abastecimento e da Reforma Agrária, 1993. 